\title{
Chapter 1 \\ Simplified Analytical/Mechanical \\ Procedure for Post-earthquake Safety \\ Evaluation and Loss Assessment of Buildings
}

\section{S. Pampanin}

\begin{abstract}
The crucial need to develop and implement simple and cost-effective repair and retrofit strategies and solutions for existing structures has been once again emphasized, if at all needed, by the recent catastrophic earthquake events. The significant socio-economic impacts of the Canterbury earthquakes sequence in 2010-2011 as well as of the "series" of independent events within few years in Italy (L'Aquila 2009; Emilia 2012; Central Italy 2016) have triggered a stepchange in the high-level approach towards the implementation of seismic risk reduction, introducing either a mandatory enforcement or significant financial incentives for a national-wide program to assess (and reduce by remedial intervention) the seismic vulnerability/capacity of the whole (non-dwelling) building stock, including safety and expected repairing costs (direct economic losses). This chapter provides an overview of the motivations, challenges and (possible) solutions for such a complex and delicate task with the intent to stimulate awareness, discussion and synergetic actions within the wider international community. Particular focus will be given to the development and on-going continuos refinement of a simplified analyticalmechanical methodology_referred to as SLaMA (Simple Lateral Mechanism Analysis) method-as part of a proposed integrated methodology for either pre- and postearthquake safety evaluation and loss assessment of buildings, in order to support the engineering community and stakeholders through the various steps of the decision making process of risk (assessment and) reduction.
\end{abstract}

\footnotetext{
S. Pampanin (凶)

Full Professor, Department of Structural and Geotechnical Engineering, Sapienza University of Rome, Via Eudossiana 18, 00184 Rome, Italy

e-mail: stefano.pampanin@uniroma1.it
}

Adjunct Professor, Department of Civil and Natural Resources Engineering, University of Canterbury, Christchurch, New Zealand

S. Akkar et al. (eds.), Advances in Assessment and Modeling of Earthquake Loss, Springer Tracts in Civil Engineering,

https://doi.org/10.1007/978-3-030-68813-4_1 


\subsection{Introduction}

The urgency of a medium-long-term plan for seismic retrofit and risk reduction strategy at a national scale is becoming increasingly evident in most of the seismicprone countries worldwide.

With no doubt the assessment of the seismic vulnerability of existing buildings and the definition of appropriate solutions-i.e. structurally effective, easy to apply, cost-effective, possibly reversible and respectful of the architectural, heritage and cultural conservation requirements-hide a level of significantly higher complexity than designing new structures.

Even more when dealing with damaged buildings in the aftermath of an earthquake event: some of the most controversial issues in the recent earthquakes have in fact been the evident difficulty and lack of knowledge/guidelines in terms of: (a) evaluation of the residual capacity of a damaged building to sustain subsequent aftershocks; (b) selection and implementation of a set of reliable repairing techniques to bring back the structure "at least" to its conditions before the earthquake; and (c) capacity to predict the cost (or cost-effectiveness) of such a repair intervention, when compared to fully replacement costs and accounting for potential aftershock in the near future.

Moreover, as if the technical complexity was not a sufficient deterrent, the constraint of economic resources for a national scale implementation and the lack of a prioritization plan, based on risk considerations, loss assessment and cost-benefit analyses, are often referred to, or blamed as, primary obstacles to the practical implementation of such a broad and ambitious project. Yet, studies and comparative evaluations of the social-economic effectiveness of a seismic prevention strategy, when opposite to a post-event reaction/repair/reconstruction approach, clearly show its significant long-term and national benefits.

To tackle this delicate issue, it is necessary to improve and standardize the tools and procedures ('protocols') for the 'diagnosis' and 'prognosis' of the seismic vulnerability and of the expected performance of existing buildings, in order to estimate, prior to the event, as well as assess, in the aftermath of the earthquake, both the seismic safety as well as the socio-economic impact/consequences/losses.

Such procedures should be based on state-of-the-art but simplified methodologies - preferably analytical rather than numerical approaches-that could highlight the structural weaknesses of the building system, while ensuring consistency of results and proper level of independently from the operators. Similarly, suitable 'therapeutic pathways', i.e. appropriate repair/retrofit strategies, can be defined by comparing alternative options through a cost-benefit approach.

This chapter provides an overview of recent developments at international level in terms of methodologies and regulatory approaches as well as 'diagnosis' protocols and procedures for either pre- and post-damage of existing buildings, with particular attention to (a) the features of the recently adopted national plan for seismic risk reduction in New Zealand, following the recent and devastating 2010-2011 earthquake sequence in Christchurch, (b) the associated seismic assessment and rating methodology, incorporated in the New Zealand Guidelines on Seismic Assessment 
of Existing Building (NZSEE 2017) and endorsed by the Mnistry of Business Innovation and Employment and (c) the Seismic Risk Classification guidelines introduced in Italy in 2017 (ITA 2017), following two decades of severe earthquake events, and including both safety and economic-loss considerations.

Building on, and merging, these recent developments, an integrated methodology for the evaluation and assessment of either pre-earthquake as well as post-earthquake safety and losses, based on a simplified analytical-mechanical approach, referrred to as SLaMA (Simple Lateral Mechanism Analysis), is presented.

\subsection{Seismic Risk Reduction Policies}

\subsubsection{The New Zealand Passive Approach-"Before"}

Before the dramatic Canterbury Earthquake Sequence 2010-2011 in New Zealand, the preparation and implementation of seismic risk reduction policies were delegated to the local territorial authorities (TAs). With the exception of the capital city of Wellington, who had initiated an active approach with a trial assessment on a large sample of buildings, the typical policy adopted by the TAs was based on a 'passive' approach, similar to the general trend at international level in major seismic-prone countries. According to such policy, there was no mandatory requirement to assess the seismic vulnerability and capacity of existing private or public buildings unless the owner aimed for a change of use, increase in volume or structural alteration. The Building ACT (2004) had defined a minimum level of 'seismic safety' for a building corresponding to the exceedance of its 'ultimate capacity' under a 'moderate' Earthquake, the latter being defined as "an earthquake that would generate shaking at the site of the building that is of the same duration as, but that is one-third as strong as, the earthquake shaking (determined by normal measures of acceleration, velocity and displacement) that would be used to design a new building".

Translating these legal and policy-making wordsmithing into engineering practical procedures, within the NZSEE2006 Guidelines on "Assessment and Improvement of the Seismic Performance of Buildings in Earthquakes" (NZSEE 2006), the 'ultimate capacity' was taken as the Ultimate Limit State (or Life Safety, using a common international nomenclature) and the 'moderate earthquake' was taken as the seismic demand (spectral intensity) corresponding to $33 \%$ of the design intensity for a new building (i.e. 500 years return period for an ordinary structure with a life span of 50 years). A Safety Index referred to as \%NBS (\% New Building Standard), or Capacity/Demand ratio_-given by the ratio of the Capacity at Ultimate Limite State of the existing structure versus the Demand for a new structure-was introduced in the verification method. A building 'scoring' less than 33\% NBS would be identified as an Earthquake Prone Building (EPB) with a seismic Rating (or class) "D”, thus considered unacceptable (Fig. 1.1). 


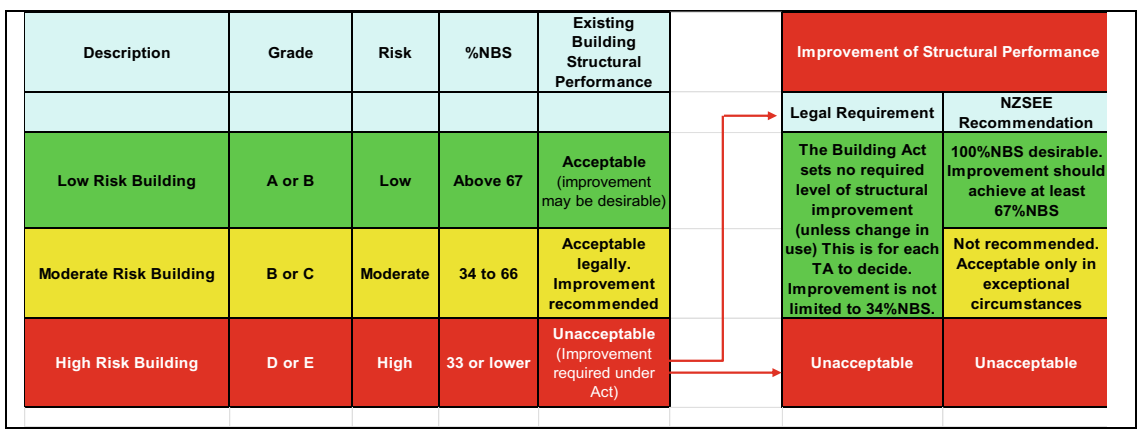

Fig. 1.1 NZSEE2017 guidelines-matrix of seismic rating, vulnerability classes and required (Building ACT 2004) or recommended remedial actions

At this stage, the owner of an Earthquake Prone Building (EPB) was obliged to retrofit/strengthen the building (even just) above the minimum threshold of $33 \%$ NBS - at his/her expenses - but only if he/she intended to proceed with the alteration/change of use of the buildings. A potential loophole of such a passive approach- potentially carrying legal liability or at least ethical responsibility-was thus that the owner, either private or public, could opt out and maintain the status quo ("no action" or "do-nothing" approach), in spite of being aware of the high seismic risk of the building and thus of the life-safety risk for the occupants.

It is also important to note here that, as shown in the same figure/table with the same green band, the New Zealand Society for Earthquake Engineering, from its first document NZSEE2006 and furthermore in the significantly revised version NZSEE2017, has been strongly recommending to target $67 \%$ NBS as a minimum objective of the retrofit and, as practically as possible, a higher value.

\subsubsection{Relationship Between \%NBS and Collapse Risk}

Already the original NZSEE2006 Guidelines were qualitatively discussing the approximate correlation between the \%NBS of an existing building and its actual (predicted and approximate) relative seismic risk, intended as a multiplier of the Life Safety (or Collapse) probability of the building when compared to that of an 'equivalent' new building.

Notably, as shown in Fig. 1.2, when decreasing the \% NBS-which depends on multiple factors such as: (a) the building material, i.e. unreinforced masonry versus concrete or steel; (b) the structural system, i.e. frame, walls, dual system and (c) the structural details and the design philosophy related to the requirements/recommendations of the design code/guidelines of the time-a more-thanlinear increase of the collapse risk, when compared to a newly designed building design, would be expected. Furthermore, the range (dispersion) of the expected Relative Risk would significantly increase, reflecting the higher uncertainties related to 


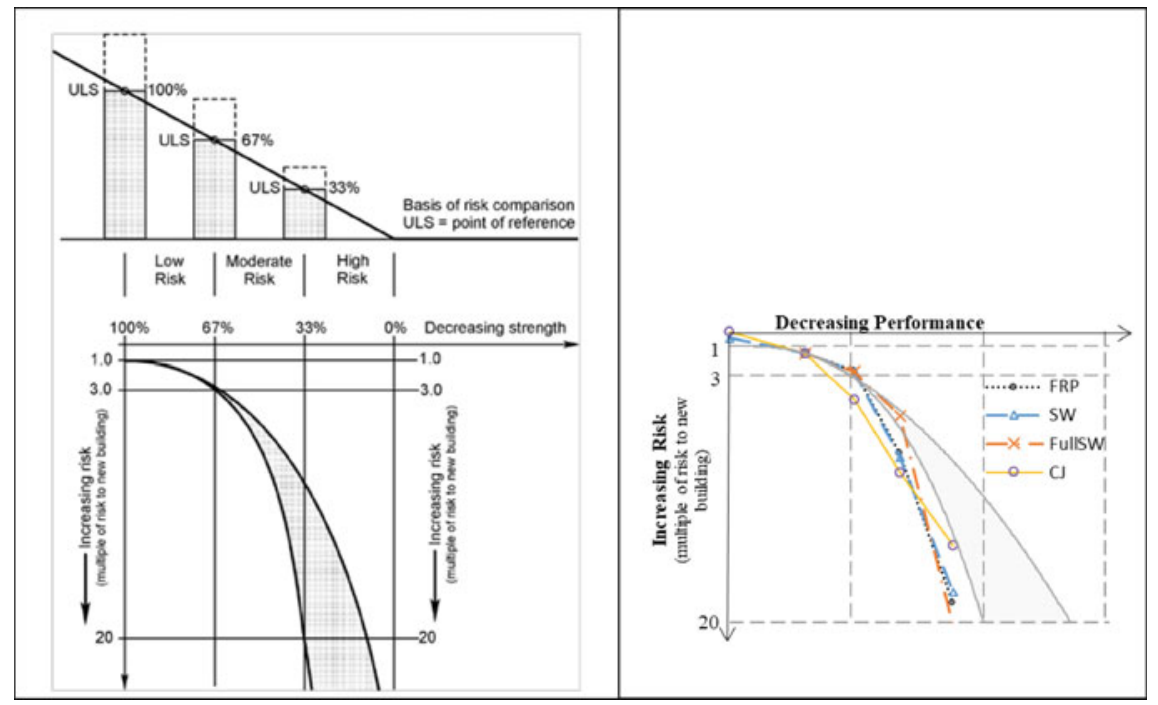

Fig. 1.2 Left: NZSEE2017 - Qualitative (approximate) correlation between the \%NBS (Capacity/Demand ratio) and the Relative Seismic Risk (Life Safety) compared to a new building (or a building retrofitted to a \%NBS). Right : example of risk reduction following the implementation of alternative retrofit strategies (Fiber Reinfroced Polymers, FRP; Selecrive Weakening, SW; Full Selective Weakening, FW; Column Jacketing, CJ; from (Ligabue et al. 2015).

the seismic response of building designed according to obsolete knowledge/codes and poor details.

Such considerations can suggest interesting corollaries-as confirmed by recent numerical studies (Ligabue et al. 2015, Fig. 1.2 right)—in terms of seismic retrofit strategies. Starting from a high risk building with a \%NBS below $33 \%$ and a seismic rating $\mathrm{D}$ or $\mathrm{E}$, small but focused local retrofit interventions, able to moderately improve the \%NBS within the same class, could lead to a substantial and proportionally more significant reduction of the seismic risk in terms of Life Safety, whilst improving the overall reliability of the seismic response of the building, i.e. reducing the dispersion and uncertainties related to the behaviour of the structures.

\subsubsection{The New National Plan for Seismic Risk Reduction in New Zealand}

In the aftermath of the Canterbury Earthquake Sequence and following the recommendation from the 'Canterbury Earthquake Royal Commission of inquiry' (CERC 2012), the New Zealand Government decided to radically 'change gear' in terms of seismic risk reduction strategies and policies, moving from a 'passive' approach to an 'active' and mandatory one at a national level. 
The Building (Earthquake Prone Buildings) Amendment Act 2016 (2016), taking effects from 1 July 2017, introduced major step-changes to the way earthquake prone buildings are identified and managed under the Building Act, under a new national plan for managing buildings in New Zealand. Explicit intent of this national policy framework is to ensure a more consistent and homogeneous approach across the country in the management of the seismic risk for private and public buildings, trying to achieve a balance between (1) Life safety, (2) Cost of strengthening/retrofitting or removing buildings and (3) Considerations on Heritage Buildings.

The new Building Act categorises New Zealand into three seismic risk áreashigh, medium and low-corresponding to peak ground acceleration, PGA, or Z (seismic coefficient in accordance to the NZS1170.5 Loading Standard (2004) of PGA $<0.15 ; 0.15 \leq$ PGA $<0.3 ;$ PGA $\geq 0.30$, respectively, as shown in Fig. 1.3. Timeframes are set for each seismic areas for identifying earthquake-prone buildings (seismic assessment, within 5-10-15 years respectively) and taking action on them (retrofit or demolition, 15-25-25 years, respectively). A special category of 'priority buildings' is introduced, in high and medium seismic areas, to recognize buildings that are considered higher risk because of their construction, type, use or location. For this category, the timeframe for both assessment and, if need be, remediation is half the time allowed for other buildings in the area.

Important to highlight that the owners should bear the costs for these seismic evaluation and remediation actions. No specific financial incentives, as for example implemented in the recent Italian Guidelines for the classification of the seismic risk (Giovinazzi and Pampanin 2017), were introduced at that stage nor were envisaged in the near future for ordinary buildings in New Zealand. Discussions are taking place in some local municipalities to address the issue of preservation of heritage buildings, often owned by private people, but with a significant yet non-tangible value for the overall community.
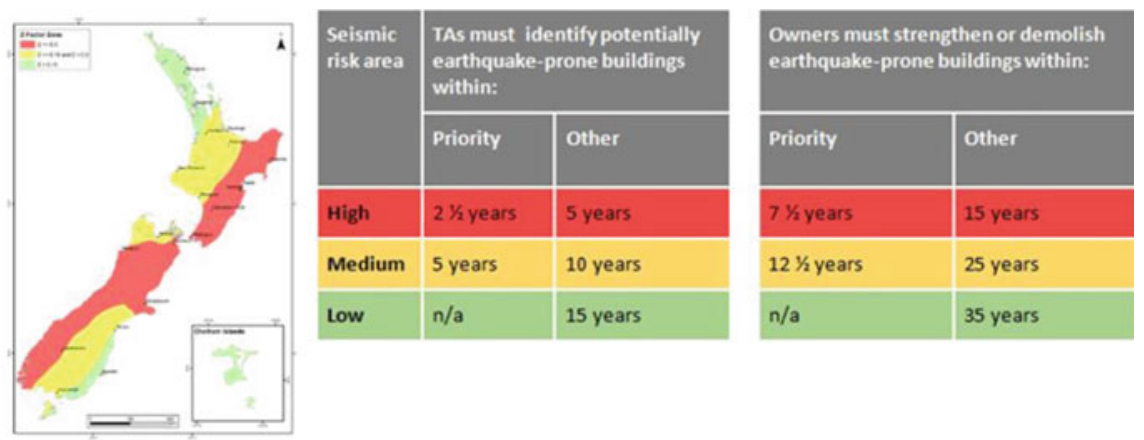

Fig. 1.3 National risk reduction plan in New Zealand (taking effect from 1 July 2017): timeframe for the identification and the remediation (strengthening/retrofit or demolition) of Earthquake Prone Buildings depending on the level of seismicity (low-medium-high seismic areas-PGA $<0.15 \mathrm{~g}$; $0.15 \mathrm{~g} \leq \mathrm{PGA}<0.3 \mathrm{~g} ;$ PGA $\geq 0.30 \mathrm{~g}$ )-MBIE 2016 


\subsubsection{The NZSEE2017 Guidelines on Seismic Assessment of Existing Buildings}

As part of the new national risk reduction plan, the Ministry for Business, Innovation and Economy (MBIE) was in charge of the development of new guidelines for the Seismic Assessment (and Rating) of Existing Buildings, with particular focus on a consistent procedure to be used on a daily based by practitioner engineer and Territorial Authorities (TAs) to identify Earthquake Prone Buildings. Following a major multi-year effort of the selected committee, which actually started in 2014 following the CERC Recommendations, the older version of the NZSEE2006 was significantly rewritten and integrated with the more recent lessons and know-how from earthquake events, as well as from the national and international state-of-art and latest research findings.

As it stands, the new NZSEE2017 guidelines "The Seismic Assessment of Existing Buildings" represents the key tool for the actual implementation of the national risk reduction plan. An overview of the motivations, scope and methodology can be found in (Pampanin 2017), with design example and numerical studies on different Structural systems in (Vecchio et al. 2017, 2018) and (Gentile et al. 2019a, b, c, d), respectively.

In particular, the NZSEE2017 guidelines place significant effort and focus to the use of a simplified analytical procedure - referred to as SLaMA Approach, acronym for 'Simple Lateral Mechanism Analysis', for the evaluation of the capacities (in terms of combination of forces and displacements) of structural elements, connections, subassemblies and seismic-resistant systems, as a fundamental starting point for any vulnerability/safety study and thus prior to any numerical modelling or more sophisticated (but not necessarily more accurate) analysis.

It is an explicit approach to revamp the importance of looking at the big picture with an holistic view, of understanding and controlling the design and thus restore the crucial role of the structural/geotechnical engineers, today often and unfortunately relegated, at least in the views of non-technical customers and decision makers, to the mere role of 'structural calculators'.

Furthermore, as the SLaMa approach allows to highlight the critical structural weaknesses, to evaluate the hierarchy of strength and the sequence of events/mechanisms, it naturally forms the fundamental first step ('diagnosis') to define appropriate retrofit strategies and technique ( 'prognosis' and 'therapeutic protocol') and critically compare alternative options by considering the overall performance improvement under different earthquake intensity levels, and the achievable reduction of the direct and indirect losses, evaluated through Expected Annual Losses (EAL) or similar indicators. 


\subsubsection{The New Italian Guidelines 2017 Seismic Risk Classification and Financial Incentives}

While Life Safety is, and must remain, the highest priority and target of seismic risk reduction policies, the crucial need to consider, assess and, most importantly, reduce the significant direct and indirect losses associated to the damage of structures and infrastructures has become more and more evident. At international level, such considerations have been recently implemented into a regulatory proactive approach for seismic risk reduction based on financial incentives in Italy. Following the significant socio-economic impacts suffered by Italy in the past 20 years following a series of events dislocated across the whole nation (Umbria-Marche 1997; Molise 2002; L'Aquila 2009; Emilia 2012, Central Italy 2016), the government has recently issued (effective from 1 March 2017) a new law providing financial incentives to seismically retrofit privately owned buildings with tax deductions (recoverable in five years, but with the possibility of transferring this tax credit to third parties such as financial institutes, contractors etc.) of $75-85 \%$ of the cost of the retrofit intervention) depending on level of improvement in terms of Risk Class (grades A-G) from the status quo (ITA 2017).

Worth noting that the seismic Risk Classification of the ITA2017 guidelines is based on the combination of:

(1) a Safety Index $I S$ - $V$, (equivalent to the \%NBS used in the NZSEE2006 and now NZSEE2017 guidelines) defined as the capacity/demand ratio at Life Safety limit state and

(2) the Expected Annual Losses, EAL (Perdita Annua Media, PAM, in Italian) able to take into account the various performances under different intensity levels and limit states

The Risk Class for a building under analysis will be identified as the minimum of the risk classes determined based on the IS-V (=\%NBS) and on the PAM (=EAL) assessed grades, i.e. Risk Class $=$ MIN (IS-V/\%NBS Class; PAM/EAL Class).

Tables 1.1 and 1.2 illustrate the two Risk Class and Grades matrixes according to the NZSEE2017 and ITA2017 Guidelines, respectively. It is worth noting that the

Table 1.1 NZSEE2017

Matrix-\% NBS, Grade,

Level of Risk, Remedial

Actions (modified after per

Building Act 2016)

\begin{tabular}{|c|c|c|c|}
\hline$\%$ NBS & Grade & Life risk & Safety prescription \\
\hline$>100$ & $\mathrm{~A}^{+} \mathrm{NBS}$ & Low & Acceptable $^{1}$ \\
\hline $80-100$ & $\mathrm{~A}_{\mathrm{NBS}}$ & Low & Acceptable $^{1}$ \\
\hline $67-79$ & $\mathrm{~B}_{\mathrm{NBS}}$ & Low/medium & Acceptable $^{1}$ \\
\hline $34-66$ & $\mathrm{C}_{\mathrm{NBS}}$ & Medium & Acceptable $^{2}$ \\
\hline $20-34$ & $\mathrm{D}_{\mathrm{NBS}}$ & High & Legally \\
\hline$<20$ & $\mathrm{E}_{\mathrm{NBS}}$ & Very high & Unacceptable $^{3}$ \\
\hline
\end{tabular}


Table 1.2 ITA2017 Matrix—safety index IS-V (=\% NBS), risk classes, grades, EAL classes and ranges

\begin{tabular}{l|l|l|l}
\hline IS-V class & IS-V ranges & EAL class & EAL ranges \\
\hline $\mathrm{A}^{+}$IS-V & IS-V $>100$ & $\mathrm{~A}^{+}{ }_{\text {PAM }}$ & EAL $\leq 0.5 \%$ \\
\hline $\mathrm{A}_{\text {IS-V }}$ & $80 \% \leq \mathrm{IS}-\mathrm{V}<100 \%$ & $\mathrm{~A}_{\text {PAM }}$ & $0.5 \%<\mathrm{EAL} \leq 1.0 \%$ \\
\hline $\mathrm{B}_{\text {IS-V }}$ & $60 \% \leq \mathrm{IS}-\mathrm{V}<80 \%$ & $\mathrm{~B}_{\text {PAM }}$ & $1.0 \%<\mathrm{EAL} \leq 1.5 \%$ \\
\hline $\mathrm{C}_{\text {IS-V }}$ & $45 \% \leq \mathrm{IS}-\mathrm{V}<60 \%$ & $\mathrm{C}_{\text {PAM }}$ & $1.5 \%<\mathrm{EAL} \leq 2.5 \%$ \\
\hline $\mathrm{D}_{\text {IS-V }}$ & $30 \% \leq \mathrm{IS}-\mathrm{V}<45 \%$ & $\mathrm{D}_{\text {PAM }}$ & $2.5 \%<\mathrm{EAL} \leq 3.5 \%$ \\
\hline $\mathrm{E}_{\mathrm{IS}-\mathrm{V}}$ & $15 \% \leq \mathrm{IS}-\mathrm{V}<30 \%$ & EPAM $_{\text {PA }}$ & $3.5 \%<\mathrm{EAL} \leq 4.5 \%$ \\
\hline $\mathrm{F}_{\text {IS-V }}$ & $\mathrm{IS}-\mathrm{V}<15 \%$ & $\mathrm{~F}_{\text {PAM }}$ & $4.5 \%<\mathrm{EAL} \leq 7.5 \%$ \\
\hline- & - & GPAM & $7.5 \% \leq \mathrm{EAL}$ \\
\hline
\end{tabular}

ranges of $\% \mathrm{NBS}$ or IS-V adopted to define the Grades or Risk Class are very similar. On the lower classes C-D-E-F (poorer performance) the ITA2017 guidelines have a more detailed classification (and one more class G) while NZSEE2017 code tends to use larger ranges, in recognition of the increasing level of uncertainties associated to the calculation of such index for older and poorly detailed structures.

\subsection{The SLaMA Analytical-Mechanical Assessment Procedure}

In line with the Park, Paulay and Priestley 'School', in the new NZSEE2017 guidelines, and specifically at the Detailed Seismic Assessment (DSA) level (Tier 2), particular attention has been given to the development of an advanced, reliable while simplified methodology, referred to as SLaMA (Simple Lateral Mechanism Analysis), based on an analytical and mechanical approach, i.e. basically "by hand" or using a spreadsheet, rather than and prior to a numerical (i.e. finite element and computer-based).

The SLaMA procedure for the assessment of the seismic vulnerability/safety and seismic rating (Fig. 1.4) develops from the identifications of the expected critical structural weaknesses through the evaluation of:

- Step 1(a): the flexural and shear capacity (in terms of both forces and displacements) of the structural elements;

- Step 1(b): the hierarchy of strength and sequence of events of the connections and beam-column subassemblies [according to Pampanin and Bolognini (2007)]

- Step 1(c): the local and global collapse mechanisms of the seismic-resisting systems and, finally, the global capacity curve 


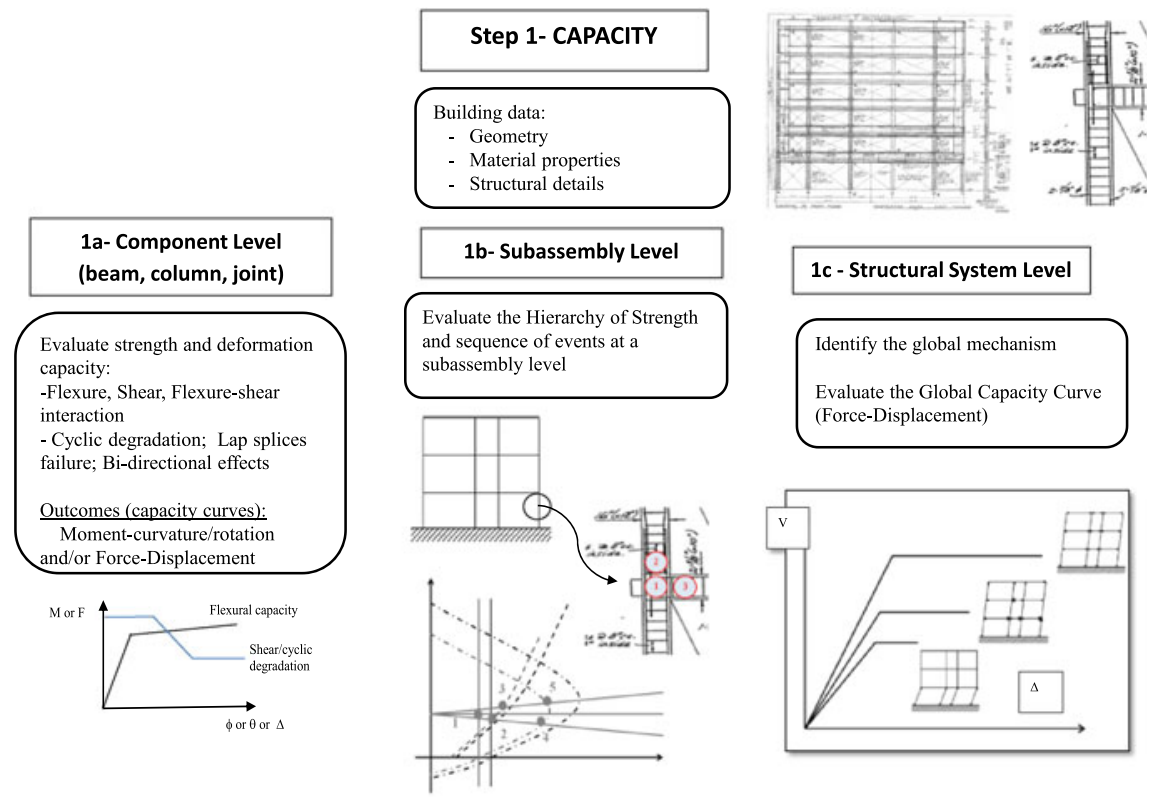

Fig. 1.4 Key steps of the SLaMa analytical procedure according to the NZSEE2017 guidelines (Pampanin 2017) to derive the capacity curve of a building: 1(a) evaluation of the strength and deformation capacity for flexure and shear of structural components; $\mathbf{1}(\mathbf{b})$ evaluation of the hierarchy of strength and sequence of events in the beam-column systems and $\mathbf{1}(\mathbf{c})$ identification of the global mechanism and evaluation of the global force-displacement capacity curve

By comparing the Capacity curve of the structure (in terms of analytical force-displacement non-linear curve) and the Demand (in terms of accelerationdisplacement response spectra, ADRS, in line with the capacity spectrum method or similar approaches), the expected performance of the building under different level of shaking intensity, i.e. earthquake return periods, can be evaluated with a relatively simple approach and good level of approximations (Fig. 1.5).

More specifically, given the NZSEE2017 key focus on Life Safety, such an analytical approach allows to evaluate the level of 'relative' (Life) Safety when compared to a newly built structure, by means of the \%NBS index (New Building Standard, a capacity-demand ratio) and to associate a seismic risk rating or class (from A to $\mathrm{E}$, with indicative ranges of collapse probabilities when compared to a brand-new structure).

As demonstrated in a series of analytical-numerical comparisons (Bianchi et al. 2019; Gentile et al. 2019a, b, c, d; Vecchio et al. 2017, 2018), the SLaMa analytical procedure provides quite reliable results, particularly satisfactory when considering the simplicity of the method.

Refinement of the results can be obtained by preparing a non-linear numerical lumped plasticity model, 'informed' by the hierarchy of strength and local mechanism predicted by SLaMA, and running pushover or time history analyses. 


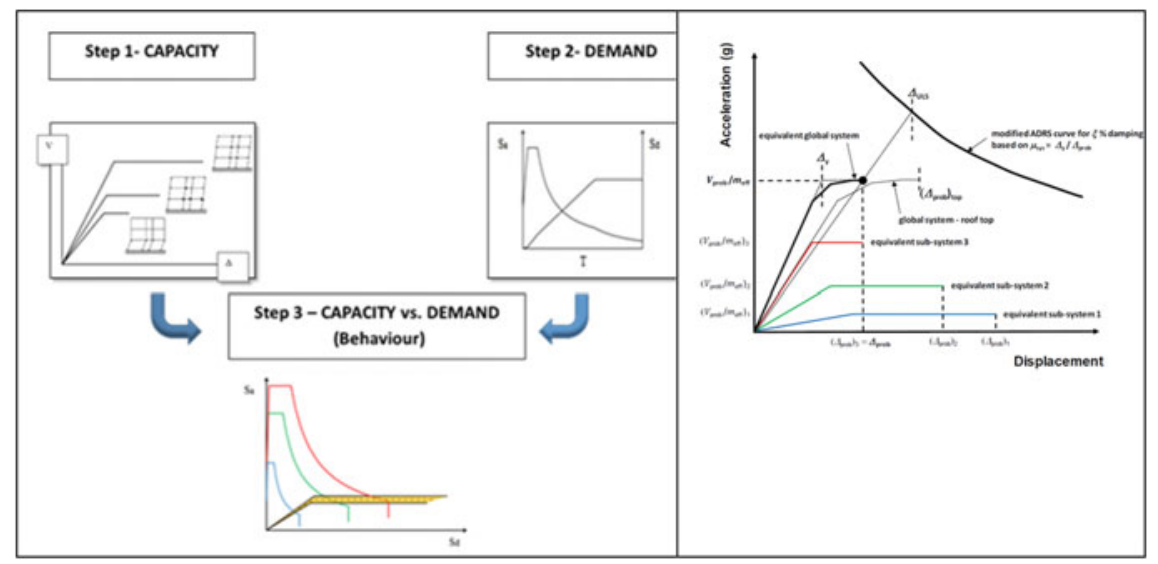

Fig. 1.5 Left: Evaluation of the seismic performance at different level of intensity by intersecting the Capacity (force-displacement) curve with the Demand (ADRS-Acceleration-Displacement Response Spectra). Right: evaluation of the \%NBS as Capacity/Demand ratio, (modified after NZSEE2006-2017)

\subsubsection{Selection of Retrofit Strategies and Techniques}

The SLaMa method can be used for a relatively quick yet reliable - when compared to what required by more complex and detailed non-linear pushover and time-history analyses - estimation of the expected behavior and performance of the building (or classes of buildings) before and after a retrofit/strengthening intervention, thus becoming a fundamental supporting tool for the implementation of a medium-long term strategy of seismic-risk reduction at national scale.

In fact, as part of the analytical evaluation of the force-displacement capacity curve of the system, the sequence of local and global mechanisms can be captured (i.e. what happens at what stage). More specifically, as shown in Fig. 1.6, the values of chord rotations, interstorey drifts, (top floor or effective height) displacements corresponding to each event (as the achievement of ULS shear or flexure in a beam, column or joint) can be identified and clearly visualized in a Force-Displacement or ADRS domain.

\subsubsection{Quantifications of Impairment_Loss Estimation}

Following a capacity spectrum approach and thus intersecting the capacity curves with the various level of earthquake (shaking) intensities (and thus return Period $\mathrm{T}_{\mathrm{r}}$ ), it is possible to evaluate what intensity level (expressed in Peak Ground Acceleration, PGA) and thus what associated Return Period ( $\left.\mathrm{T}_{\mathrm{r}}\right)$ would correspond to the achievement of the four level of performances/damage/limit states, namely, i.e. Immediate 

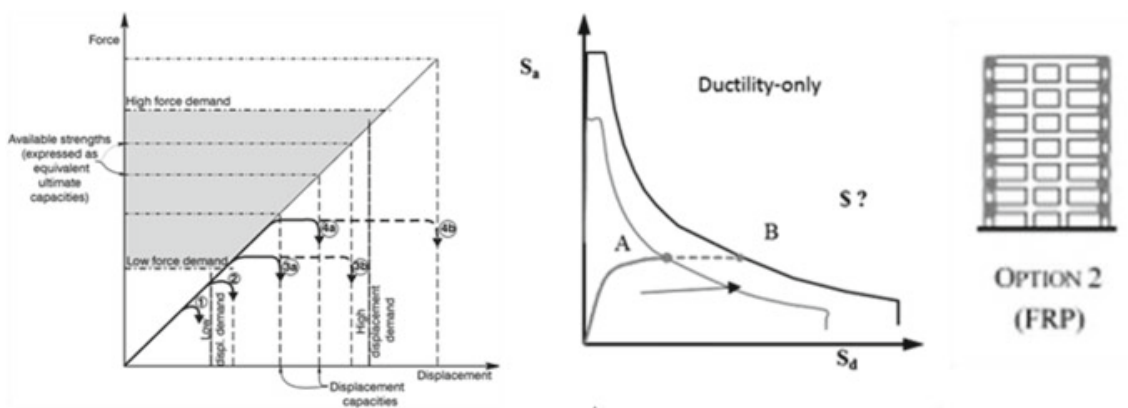

OPTION 2

(FRP)
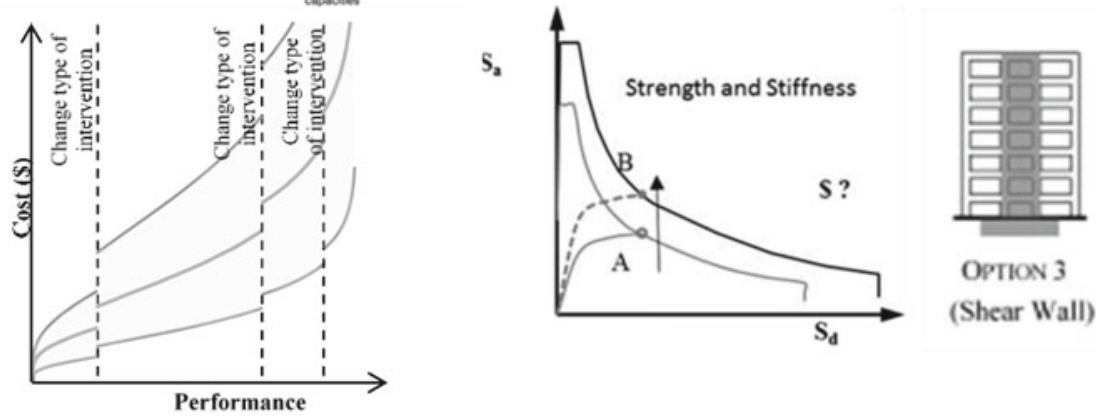

Fig. 1.6 Top Left: use of SLaMA method to capture the sequence of events/mechanisms within an analytically-derived pushover capacity curve. Right: selection of alternative retrofit strategies and techniques to achieve the targeted performance (Ligabue et al. 2015). Bottom Left: qualitative ladder-scale representing the expected cost of retrofit intervention as a function of the targeted performance or \%NBS (adapted from (Beetham 2013))

Occupancy (IO), Damage Control (DC), Life Safety (LS) and Collapse Prevention (CP), as shown in Fig. 1.7.

The relationship between PGA and Return Period depends on the local seismicity and earthquake magnitude.

The Italian Guidelines ITA2017 provide a relationship and a power coefficient (average value used herein, in principle it is function of the local seismicity and magnitude) to convert PGA into $\mathrm{T}_{\mathrm{r}}$ and vice versa, as follows:

$$
T_{r C}=T_{r D}\left(P G A_{C} / P G A_{D}\right)^{1 / 0.41}
$$

The sub indexes C and D indicate 'Capacity' and 'Demand' respectively.

In order to create a MAF versus \% Reconstruction Cost curve it is necessary to estimate the relationship between an Engineering Demand Parameter (e.g. Drift, acceleration), the Level of Damage/Limit States and the repairing costs associated to that level of damage.

Following the L'Aquila 2009 earthquake in Italy and the repair/reconstruction phases, covered by government funds through a very comprehensive scheme, a unique and detailed database of costs of reconstruction versus damage levels was 

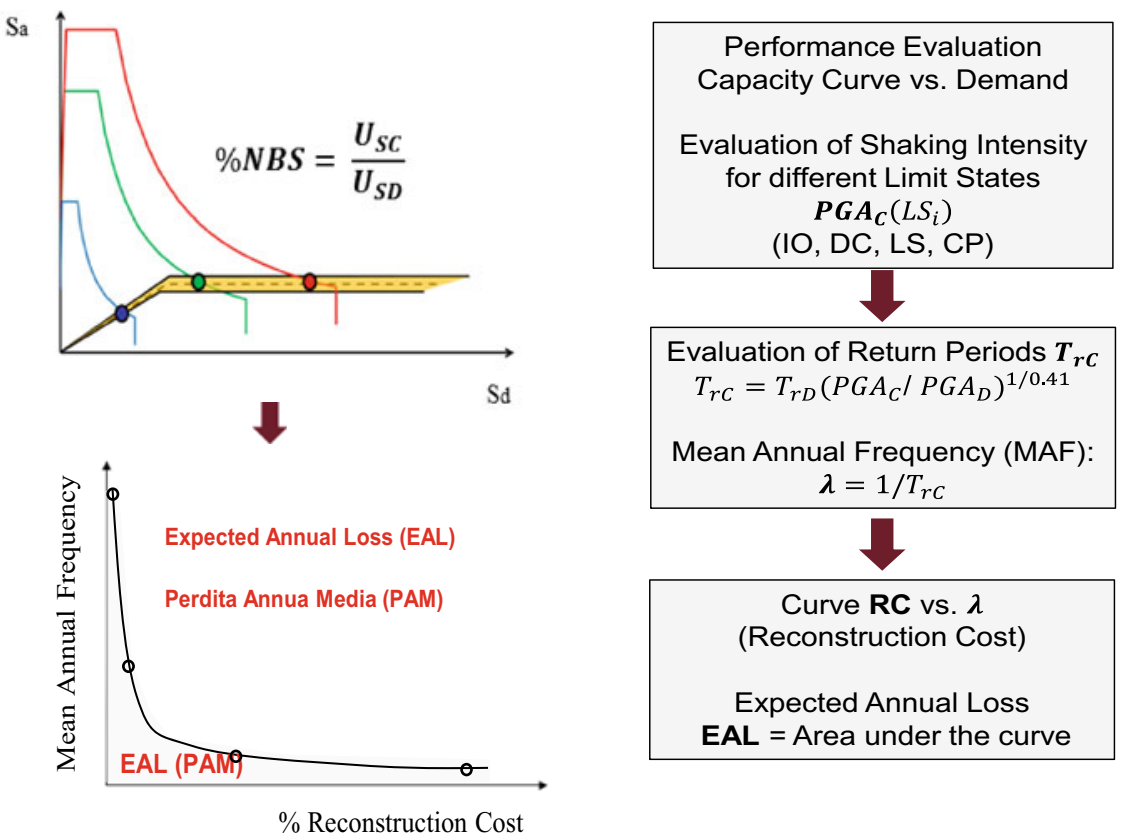

Fig. 1.7 Left: Procedure for the evaluation of the Expected Annual Loss (EAL): (1) Comparison of Capacity Curve and Demand (ADRS Spectra); (2) Evaluation of the earthquake intensity levels PGAc and associated return period Trc, corresponding to the various Limits States (IO, DC, LS, $\mathrm{CP})$. Note that in the top left-side graph only three Limit States are herein for simplicity indicated, namely IO $<\mathrm{DC}<\mathrm{LS}$ ); (3) Plot the curve Mean Annual Frequency (MAF or $\lambda=1 / \mathrm{T}_{\mathrm{rC}}$ ) versus Costs (\% reconstruction costs); (4) Evaluation of the EAL as integral (area) of this curve

collected and published in a "White Paper" or "Libro bianco" (Ludovico et al. 2017a, b).

Based on these data, a table correlating the expected (mean values of) \% Reconstruction Costs corresponding to a given limit state/observed damage was developed. As shown in Table 1.3, such correlation is a key part of the simplified procedure to evaluate the Expected Annual Loss (EAL) starting from a capacity versus demand comparison as described above.

Table 1.3 Correlation between the various Limit States (IO, DC, LS, CP) and the Reconstruction Costs according to the ITA2017 guidelines

\begin{tabular}{l|l}
\hline Limit states & Reconstruction cost RC (\%) \\
\hline Collapse or total economic loss & 100 \\
\hline Near collapse, NC & 80 \\
\hline Life safety, LS & 50 \\
\hline Damage control, DC & 15 \\
\hline Immediate operational, IO & 7 \\
\hline Zero loss & 0
\end{tabular}


The Expected Annual Loss, evaluated as the integral of the MAF versus $1 / T_{\mathrm{r}}$ curve thus the Area underneath that curve, represents the overall repairing costs so calculated/estimated during the life-span of the building divided by the 50 years (or whatever assumed as a Life-span) of the structure.

This comprehensive procedure to evaluate the Expected Annual Loss (EAL) directly from capacity curves and earthquake demands (ADRS Spectra) is well recognized within the scientific community but unique at a worldwide level in terms of regulatory provision.

Such an approach is a direct and practical response to the lessons learnt from recent earthquakes, acknowledging and highlighting the severe and significant repairing costs associated to non-structural elements (partitions/ infills, facades, ceilings, services) under 'frequent' earthquakes (i.e. minor-moderate earthquake and relatively small return period $\mathrm{T}_{\mathrm{r}}$ of 25-100 years), when compared to more 'rare' events (i.e. major-severe intensity and large return period $T_{r}$ of 500-1000 years or above) typically used as a design level reference, when thus focusing on "Life Safety" as primary Limit State.

In addition to a Time-Based loss estimation of the EAL/PAM, following a similar approach, a Scenario-Based Assessment can be used to estimate the expected level of direct economic loss, again expressed in terms of \% Reconstruction Costs under a specific event or scenario earthquake, such as:

(1) the Probable Frequent Loss (PFL) defined by Porter et al. (2004a) as the mean loss resulting from shaking with a $10 \%$ exceedance probability in 5 years (i.e. Return Period $\mathrm{Tr}=50 \mathrm{yrs}$ ) and/or

(2) the Probable Maximum Loss defined as the mean loss resulting from shaking with a $10 \%$ exceedance probability in 50 years (i.e. $\operatorname{Tr}=500 \mathrm{yrs}$ )

This latter Scenario-Based Assessment approach focuses into a specific window within the building life-time response and provides a complementary information to the EAL, which instead gives a more comprehensive, yet general and thus spread across the various limits states (and earthquake intensity), view of the overall building performance under a range of seismic excitations.

\subsection{Post-Earthquake Residual Capacity of Damaged Buildings}

In New Zealand, the Canterbury Earthquake Sequence 2010-2011 and, again few years later, the Kaikoura Earthquake 2016, similarly to the several earthquake events occurred in the past decades at international level, have highlighted the complexity and uncertainties associated with the delicate decision of repair versus demolition, due to the lack of: (a) evidence-based information/knowledge, (b) codified ad-hoc guidelines related to the (b1) evaluation of the residual capacity of a damaged 
building to sustain subsequent aftershocks and (b2) the selection and implementation of appropriate cost-effective repairing techniques to 'restore' the building to its pre-earthquake conditions (CERC 2012).

In the past years a significant research effort has been dedicated at both national and international level [US, Europe and Japan, FEMA 306 (1998), JBDPA (2015)] to gain a better understanding on the residual capacity of buildings following an earthquake event (Cuevas et al. 2014, 2015; Cuevase and Pampanin 2016, 2017; Loporcaro et al. 2014. 2017, 2018, 2019; Luco et al. 2004; Ludovico et al. 2013; Maeda 2008; Maeda and Eon-Kang 2009; Maeda et al. 2012, 2017; Mukai et al. 2017; Polese et al. 2012).

In New Zealand, the on-going findings and key outcomes of the first NZ-based project on Residual Capacity and Repairing Options [NHRP Funded, Contestable round 2012, (Pampanin et al. 2015)], have formed the basic knowledge platform of the activities of a special MBIE (Ministry of Business, Innovation and Employment) committee/working group on the topic residual capacity (Cuevas et al. 2014, 2015; Cuevas and Pampanin 2016, 2017; Loporcaro et al. 2014, 2017, 2018, 2019), leading to the development of a draft framework for the evaluation of the residual capacity of buildings (Elwood et al. 2016).

Further developments have been obtained in the recent past, suggesting to merge the benefits of different methodologies into one general analytical-mechnical approach, based on an evolution of the SLaMa method, as described and proposed in the following sections.

\subsubsection{Effects of Damage on Future Building Performance}

Provided appropriate/reliable reduced capacity curves are derived, the aforementioned general SLaMA-based approach for the assessment of seismic safety as well as the estimation of losses can be implemented for either the as-built (un-damaged), the damaged and the repaired/retrofitted (with different solutions and targeted performance) configurations, providing valuable insights to support the decision making process for various stakeholders (e.g. alternative retrofit solutions, territorial scale approach, repair versus demolition, cost/benefit analysis).

\subsubsection{General Procedure to Evaluate the Impairment of the Capacity Curves (FEMA306/307-Type)}

"Understanding the effects of damage on future building performance" has been the declared first 'objective' of the ATC-43 project (which delivered the FEMA306 and 307 guidelines) building on a thorough review (yet dated 1998) of available analysis techniques, field observations, test data, and emerging evaluation and design methodologies, and complemented by further analytical/numerical work. 
As noted in the FEMA 306 document "The quantitative evaluation of the effects of earthquake damage on structures requires the selection of a measurement parameter. Procedures in this document use change in the anticipated performance of the building during future earthquakes as the measurement parameter. This is the change due directly to effects of earthquake damage on the basic structural properties that control seismic performance. If the structural property changes are estimated, the corresponding change in future performance can also be estimated".

The quantitative procedure proposed to assess and compare the relative performance of the building in its pre-damage, post-damage and repaired state relies upon non-linear static analysis techniques (i.e. capacity spectrum method), thus fully consistent with, and achievable with, the SLAMa Method.

The Capacity Curves of the overall structures can be derived for the as-built, the damaged or repaired level, starting from the capacity curve of a component level (see Fig. 1.8) and depending on:

(a) component type (wall, pier, spandrel, coupling beam);

(b) extent of damage (minor-moderate-severe) and

(c) mode of damage/behaviour (ductile vs. brittle)

The effects of damage on the component backbone curve behaviour are taken into account by means of modification/reduction factors of stiffness $(\lambda K)$, strength $(\lambda Q)$, deformation capacity $(\lambda D)$ and Residual Drift $(\mathrm{RD})$, calibrated numerically and experimentally.

The values of these modification/reduction factors, for each component of the structural system, depend on the type of damage, according to the behaviour mode (ductile vs. brittle, flexure vs. shear prone) and the severity of damage (insignificant, slight, moderate, severe, extreme).

The FEMA 306 and 307 Guidelines provides a component Damage Classification Guide in the form of table/charts/schetces to assist the engineer with the classification of type (behaviour mode) and severity of the damage, and derive associated component modifications factors. Modifications factors are also presented to account

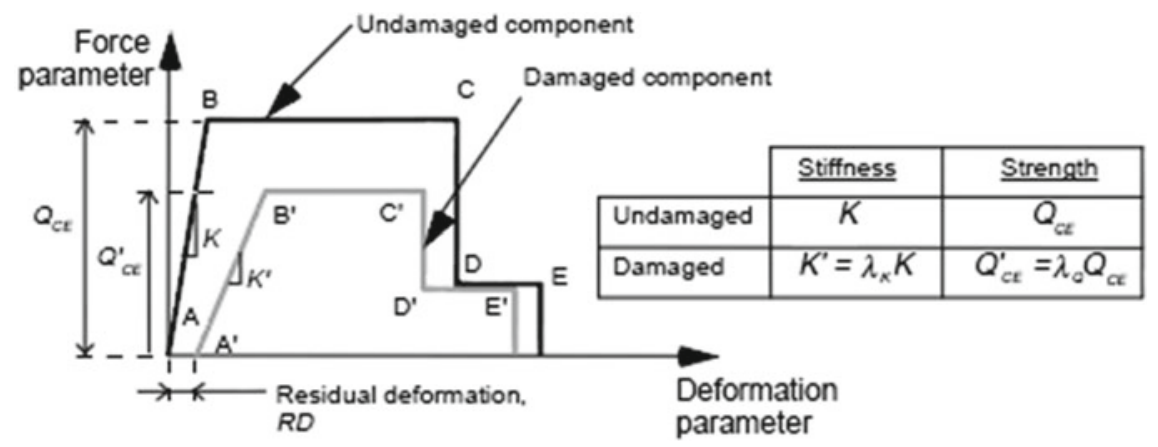

Fig. 1.8 Component modelling criteria and stiffness/strength/displacement reduction factors to account for damage (FEMA 306 (1998)) 
for repair/restoration. The documents primarily focuses on reinforced concrete and masonry wall buildings, with some insights into infills and their interactions with the surrounding frames. Limited information is available in literature for other type of structural systems and components, such as beam-column joints, beam plastic hinges, column flexural, lap splice or shear failure, floor/diaphragm behavior. Yet, recent numerical/experimental investigation in the past years have started filling the gap: in (Ludovico et al. 2013; Polese et al. 2012) modification factors were proposed to evaluate the residual capacity of non-confirming RC columns typical of the Mediterranean region; furthermore, experimental and numerical investigations on cumulating damage, residual capacity and repairing options for plastic hinges in beam-column subassemblies and beam cantilever as well as on the effects on strain hardening and low-cycle fatigue in steel rebars on the overall plastic hinge behavior and repairability have been carried out in New Zealand (Cuevas et al. 2014, 2015; Cuevas and Pampanin 2016, 2017; Elwood et al. 2016; Loporcaro et al. 2014, 2017, 2018, 2019; Luco et al. 2004; Maeda 2008; Maeda and Eon-Kang 2009; Maeda et al. 2012, 2017; Mukai et al. 2017; Pampanin et al. 2015).

\subsubsection{Proposed SLaMA-Based Procedure for Pre- and Post-Earthquake Safety Evaluation and Loss Assessment of Buildings}

Building on, a merging, the aforementioned recent development and approaches, and relying upon the current modifications factors and/or deriving specific ones (based on numerical/experimental evidences) as needed, a general and consolidated SLaMAbased method can be adopted, moving from component level to subassembly and structural system level, for the safety evaluation and loss assessment of buildings in either pre- or post-earthquake, in the as-built or retrofitted/repaired, configurations.

Figure 1.9 illustrated the steps and qualitative outcome of such loss estimation procedure when a building structure is subject to a reduction of stiffness and/or strength: for three different levels of damage, with associated levels of stiffness $\lambda K$ and strength reduction, $\lambda Q$, the area underneath the Mean Annual Frequency and the $\%$ Replacement Costs will increase, leading to three different level of Expected Annual Loss, EAL.

In general terms, depending on the assessed level of damage occurred in each component and subassembly (to be evaluated through a SLaMA force-displacement curve and ADRS spectrum), the capacity curves (momento-rotation, force-displacement) of each component can be updated with the appropriate corresponding modifications factors (in terms of stiffness, strength, deformation capacity and residual drfit/chord rotation) as provided by the most current and relevant literature. A second iteration of the SlaMA methology on the building, assuming this damage state configuration, would identify new critical structural weaknesses and possible new sequence of events, due to a modified internal hierarchy of strength, thus leading to local and global mechanism. ADRS curves would be selected to represent either the code design level, whereby a post-earthquake safety check is required, or selected levels 


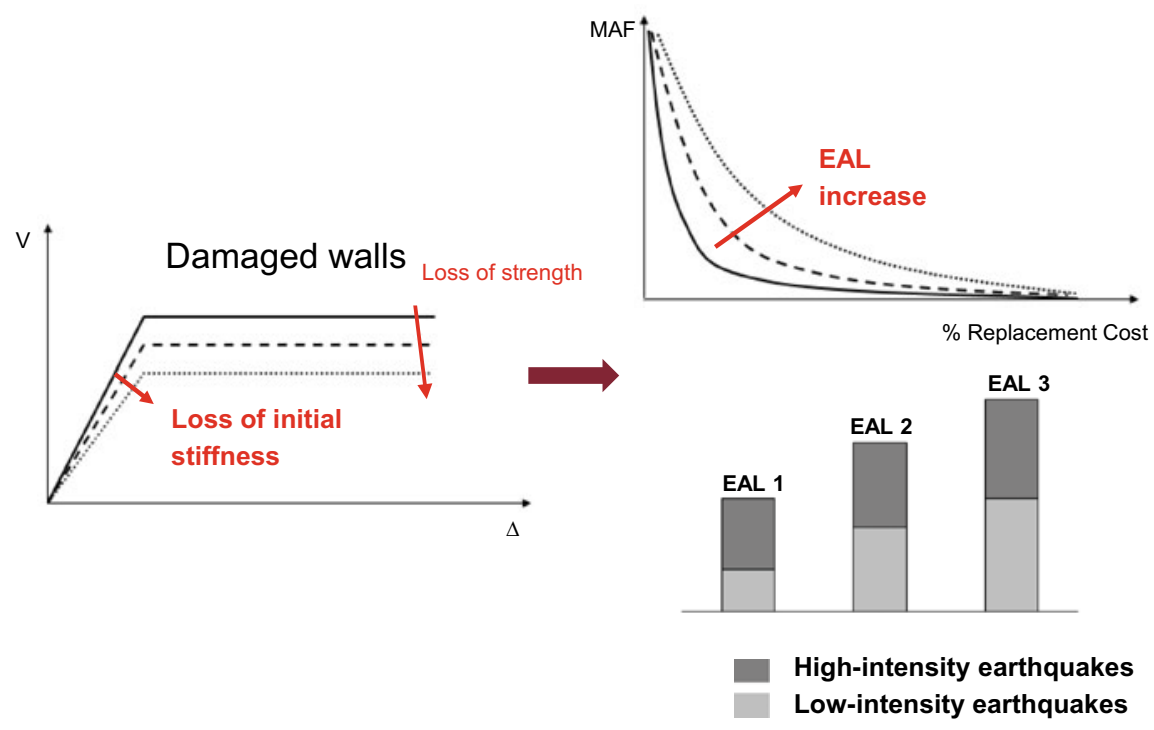

Fig. 1.9 Expected impact on performance and EAL due to loss of stiffness and/or strength as it can be evaluated following a SLaMA-based analytical-mechanical procedure

of expected aftershocks or scenerio-based events. The effects of cumulating cycles can be implemented in the procedure by means of strength degradation curves, either depending on the number of cycles and/or level of ductility at a member level, as well as bond-deterioration or stress-decay curve at a material level.

In addition to safety check and residual capacity (to withstand further aftershocks or new events) considerations, various type of losses, as previously described, e.g. EAL, PFL, or PML or other KPIs (Key Performance Indicators) such as the loss of building operability (downtime), can be evaluated, providing very valuable support to the decision making process.

As an example, it could be assessed, as done in a previous work by (Beetham 2013), that the Probable Frequent loss (PFL) is directly (possibly but not necessarily linearly) proportional to the (loss of) initial stiffness or fundamental period of vibration of the structure, as shown in Fig. 1.10 for the specific case of an existing frame building with or without alternative retrofit techniques.

Such information, related to the potential value, or loss of value, of a building in the aftermath of minor-moderate earthquake events (or in the case of aftershocks following the main shock) could be of direct interest and practical use as part of the discussion between insurance/reinsurance companies and owners or other interested parties.

Similarly, alternative retrofit or repair/retrofit strategies can be compared not only in terms of Safety Index improvement (\%NBS or IS-V) but also in terms of economical (losses) considerations as shown in Figs. 1.11 and 1.12, allowing for a more 
Fig. 1.10 Trend of Probable Frequent Loss (PFL) as a function of the \% Building Replacement Cost versus Fundamental Period of the Structures (or loss of stiffness) (Beetham 2013)
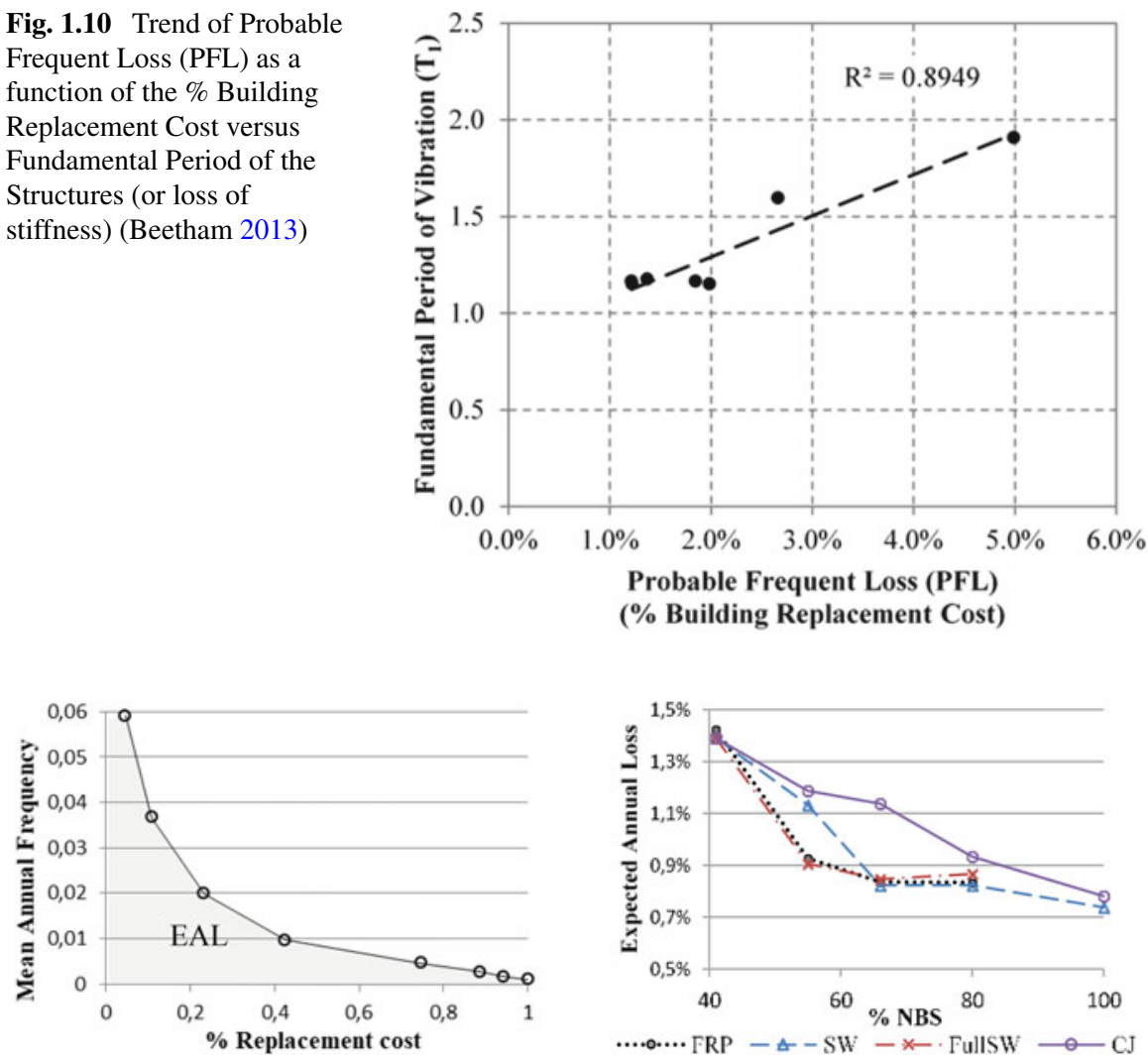

Fig. 1.11 Left: Example of Mean Annual Frequency, MAF, versus \% Replacement cost: the area under the curve represents the Expected Annual Loss, EAL (Beetham 2013); Right: Values of EAL for different retrofit strategy and \% NBS achieved (Ligabue et al. 2015)

informed decision based on (possibly and preferably multi-criteria) cost-benefit evaluation on whether or not, and to what level, it would be worth pursuing a specific retrofit/repair intervention program.

The methodology could also allow to investigate the opportunity to implement hybrid intervention at either individual building level or assets of buildings at territorial scale level, with the negotiated involvement of private, public and insurance sectors. As an example, the opted strategy could consist of a partial retrofit intervention, beared partially by the owner, as part of a self-insurance approach, and/or partially supported by direct financial incentives from the government, and/or in combination with an insurance premium rebate, adequately adjusted to recognize the enhanced and quantifiable level of protection achievable both in terms of Life Safety as well as Asset/building/Business as result of the retrofit intervention (Fig. 1.13). 


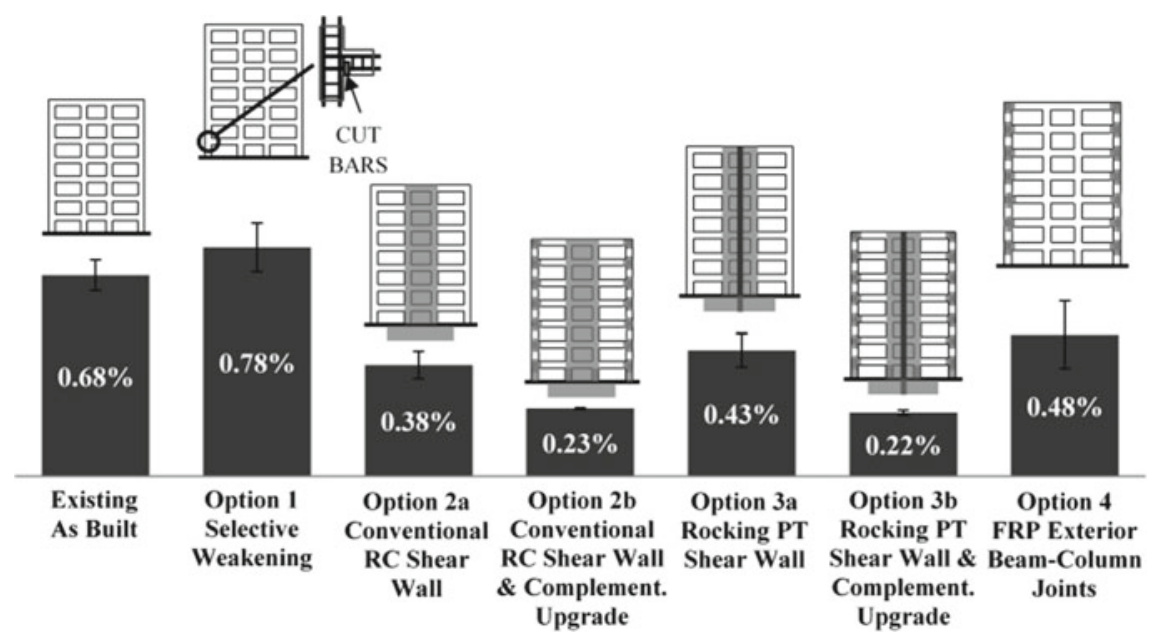

Fig. 1.12 Comparison of alternative retrofit solutions in terms of Expected annual loss (EAL) as a percentage of the building replacement cost (Beetham 2013)

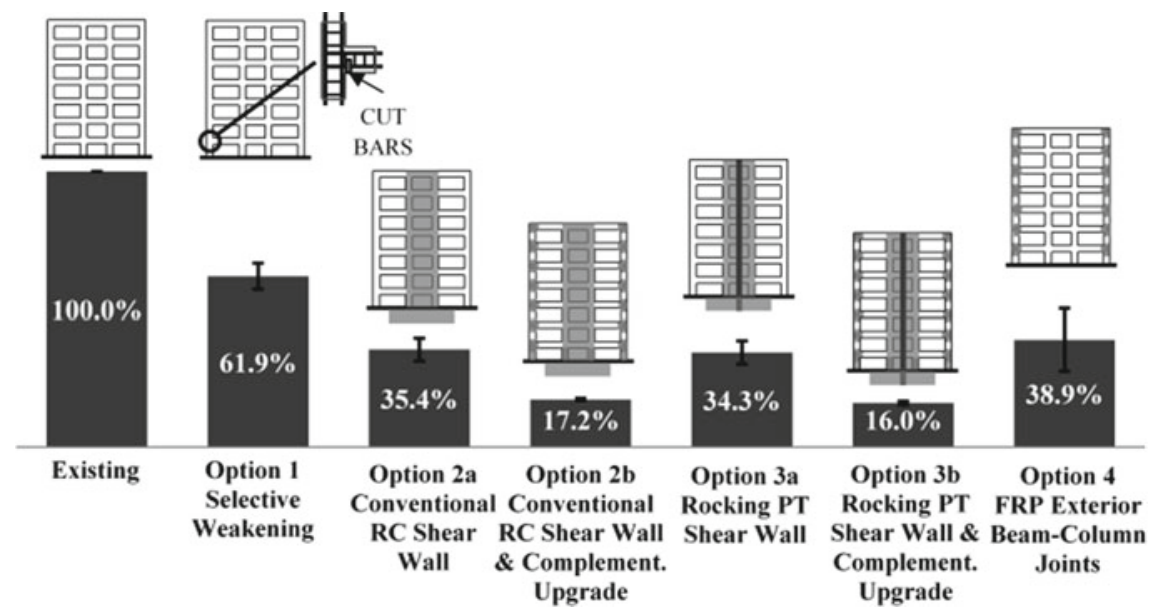

Fig. 1.13 Comparison of alternative retrofit solutions in terms of Probable maximum loss (PML) under a Design Level earthquake (500 years return period or $10 \%$ probability of exceedance in 50 years) (Beetham 2013)

\subsection{Concluding Remarks}

This chapter has provided an overview of recent developments at international level in terms of methodology and regulatory approaches as well as "diagnosis" protocols and procedures for either pre- and post-damage of existing buildings. 
Building on, and merging, these recent developments, an integrated methodology for the evaluation and assessment of either pre-earthquake as well as post-earthquake safety evaluation and loss assessment of buildings, based on a simplified analyticalmechanical approach, referrred to as SLaMA (Simple Lateral Mechanism Analysis), has been presented, in order to support the engineering community as well as the various stakeholders through the various steps of the decision making process of risk assessment and reduction.

Worth reminding that, in addition to the opportunity and value of sharing the technical content of these recent developments, the key goal remains to stimulate awareness, discussion within, as well as outside (equally if not more important) the technical community, and foster synergetic political actions for a more proactive and comprehensive implementation of seismic risk reduction programs and international level.

\section{References}

Building Act (2004) Department of building and housing-Te Tari Kaupapa Whare, ministry of economic development, New Zealand Government, Wellington, New Zealand

Beetham T (2013) Simplified displacement based performance assessment of retrofit alternatives for decision making. Master thesis, IUSS, University of Pavia, Italy

Bianchi S, Ciurlanti, J, Pampanin S (2019) A SLaMA-based analytical procedure for the Cost/Performance-based evaluation of buildings. In: Compdyn Conference, Crete, Greece

Building (Earthquake-prone Buildings) Amendment Act (2016) New Zealand Government, Wellington, New Zealand

CERC (2012) Canterbury Earthquake Royal Commission, https://canterbury.royalcommission.gov t.nz

Cuevas RA, Pampanin S (2016) Assessing the seismic residual fatigue life of reinforced concrete frame buildings: a proposed framework. In: 16th U.S.-Japan-New Zealand Workshop on the Improvement of Structural Engineering and Resiliency, Nara, Japan

Cuevas A, Pampanin S (2017) Post-seismic capacity of damaged and repaired reinforced concrete plastic hinges extracted from a real building. In: 16th WCEE, World Conference on Earthquake Engineering, Santiago, Chile, January 9-13, 2017 paper 3142

Cuevas A, Akguzel U, Pampanin S (2014) Preliminary numerical investigation on the seismic residual capacity of reinforced concrete plastic hinges. In: 2nd European Conference in Earthquake Engineering and Seismology, Istanbul 25-29 Aug

Cuevas A, Pampanin S, Carr A, Ozbolt J (2015) Seismic residual capacity of reinforced concrete frames: Part I: General Framework. Research Report UC 2015-4, Part I, Department of Civil and Natural Resources Engineering, University of Canterbury, Christchurch, New Zealand

Del Vecchio C, Gentile R, Pampanin S (2017) The simple lateral mechanism analysis (SLaMA) for the seismic performance assessment of a case study building damaged in the 2011 Christchurch Earthquake. University of Canterbury Department of Civil and Natural Resources Engineering, Research Report 2016-02, New Zealand, ISSN 1172-9511

Del Vecchio C, Gentile R, Di Ludovico M, Uva G, Pampanin S (2018) Implementation and validation of the simple lateral mechanism analysis (SLaMA) for the seismic performance assessment of a damaged case study building. J Earthq Eng Doi/full/. https://doi.org/10.1080/13632469.2018. 1483278

Di Ludovico M, Polese M, Gaetani d'Aragona M, Prota A, Manfredi G (2013) A proposal for plastic hinges modification factors for damaged RC columns. Eng Struct 51:99-112 
Di Ludovico M, Prota A, Moroni C, Manfredi G, Dolce M (2017a) Reconstruction process of damaged residential buildings outside historical centres after the L'Aquila earthquake: part I-" light damage" reconstruction. Bull Earthq Eng 15(2):667-692

Di Ludovico M, Prota A, Moroni C, Manfredi G, Dolce M (2017b) Reconstruction process of damaged residential buildings outside historical centres after the L'Aquila earthquake: part II"heavy damage" reconstruction. Bull Earthq Eng 15(2):693-729

Elwood KJ, Marder K, Pampanin S, Cuevas RA, Smith P, Cattanach A, Stannard M (2016) Draft framework for assessing residual capacity of earthquake-damaged concrete buildings. In: New Zealand Society of Earthquake Engineering Conference, Christchurch, New Zealand

FEMA 306 (1998) Evaluation of earthquake damaged concrete and masonry wall buildings: Basic procedures manual. ATC, Redwood City, CA, USA

Gentile R, Del Vecchio C, Pampanin S, Raffaele D, Uva G (2019a) Refinement and validation of the simple lateral mechanism analysis (SLaMA) procedure for RC frames. J Earthq Eng. https:// doi.org/10.1080/13632469.2018.1560377

Gentile R, Pampanin S, Raffaele D, Uva G (2019b) Non-linear analysis of RC masonry-infilled frames using the SLaMA method: Part 1-mechanical interpretation of the infill/frame interaction and formulation of the procedure. Bull Earthq Eng. https://doi.org/10.1007/s10518-019-00580-w

Gentile R, Pampanin S, Raffaele D, Uva G (2019c) Non-linear analysis of RC masonry-infilled frames using the SLaMA method: Part 2-parametric analysis and validation of the procedure. Bull Earthq Eng. https://doi.org/10.1007/s10518-019-00584-6

Gentile R, Pampanin S, Raffaele D, Uva G (2019d) Analytical seismic assessment of RC dual wall/frame systems using SLaMA: Proposal and validation. Eng Struct 188:493-505. https://doi. org/10.1016/j.engstruct.2019.03.029

Giovinazzi S, Pampanin S (2017) Simplified approaches for the seismic risk rating of reinforced concrete buildings and the selection of retrofit strategies. In: XVII ANIDIS, Italian Association for Earthquake Engineering, Pistoia, 17-22 Sept

ITA2017 (2017) Annex A to the D.M. n. 65 del 7-03-2017 "Linee Guida per la Classificazione del Rischio Sismico delle Costruzioni" (in Italian)

JBDPA (2015) Guideline for Post-earthquake Damage Evaluation and Rehabilitation (in Japanese), Japan Building Disaster Prevention Association

Ligabue V, Savoia M, Pampanin S (2015) Repairing/retrofitting vs. replacing? evaluating the costeffectiveness of alternative options to support decision making, NZSEE Conference, Rotorua

Loporcaro G, Pampanin S, Kral MV (2014) Investigating the relationship between hardness and plastic strain in reinforcing steel bars. In: NZSEE Conference, Auckland, 21-23 March, Paper No. P22

Loporcaro G, Kral M, Pampanin S (2017) Case study: application of the hardness method to estimate the residual capacity of reinforcement in an earthquake damaged building. In: 16th World Conference on Earthquake Engineering, Santiago, Chile, January 9-13, 2017, paper n. 2075

Loporcaro G, Pampanin S, Kral MV (2018) Estimating plastic strain and residual strain capacity of earthquake-damaged steel reinforcing bars. J Struct Eng 144(5). https://doi.org/https://doi.org/ 10.1061/(ASCE)ST.1943-541X.0001982

Loporcaro G, Pampanin S, Kral MV (2019) Long-term strain-ageing effects on low-carbon steel reinforcement. Const Build Mater 228. https://doi.org/https://doi.org/10.1016/j.conbuildmat. 2019.07.332

Luco N, Bazzurro P, Cornell CA (2004) Dynamic versus static computation of the residual capacity of a mainshock-damaged building to withstand an aftershock. In: 13th World Conference on Earthquake Engineering, Vancouver, Canada. Paper no. 2405

Maeda M (2008) Effect of seismic retrofit on seismic risk mitigation for reinforced concrete buildings. In: 14th World Conference on Earthquake Engineering, Beijing, China

Maeda M, Eon-Kang D (2009) Post-earthquake damage evaluation of reinforced concrete buildings. J Adv Conc Tech 7(3):327-335 
Maeda M, Nakano Y, Lee KS (2012) Post-earthquake damage evaluation for R/C buildings based on residual seismic capacity. In: Proceedings of the 13th World Conference on Earthquake Engineering, Vancouver, Canada

Maeda M, Nishida T, Matsukawa K, Murakami M (2017) Revision of guideline for postearthquake damage evaluation of reinforced concrete buildings in Japan. In: 16th World Conference on Earthquake Engineering, Santiago, Chile

MBIE (2016) - Building (Earthquake prone Buildings) Amendment Act, 2016, Ministry of Business, Innovation and Employment, NZ. Portal 'Managing Earthquake Prone Buildings', https:// www.building.govt.nz/managing-buildings/managing-earthquake-prone-buildings/

Mukai T, Kabeyasawa T, Tani M, Suwada H, Fukuyama H (2017) Residual seismic capacity of ductile RC frame with wing walls based on full-scale loading test. Bull New Zealand Soc Earthq Eng 50(44):565-573

NZS1170.5 (2004) Structural design actions. Part 5: Earthquake actions-New Zealand, Standards New Zealand, Wellington, NZ

NZSEE (2006) Assessment and improvement of the seismic performance of existing buildings, New Zealand Society of Earthquake Engineering, Wellington, https://www.nzsee.org.nz/public ations/assessment-and-improvement-of-the-structural-performance-of-buildings-in-earthquake/

NZSEE (2017) The seismic assessment of existing buildings (the guidelines), New Zealand Society of Earthquake Engineering, Wellington, Version October 2016. https://www.eq-assess.org.nz/

Pampanin S (2017) Towards the practical implementation of performance-based assessment and retrofit strategies for RC buildings: challenges and solutions. In: SMAR2017- Fourth conference on Smart Monitoring, Assessment and Rehabilitation of Structures, Keynote Lecture, 13-15 Sept, Zurich, Switzerland

Pampanin S, Bolognini, Pavese D (2007) Performance-based seismic retrofit strategy for existing reinforced concrete frame systems using FRP composites. ASCE J Comp Const, Special Issue on "Recent International Advances in FRP Research and Application in Construction 11(2):211-226. ISSN 1090-0268/2007/2-211-226

Pampanin S, Cuevas A, Kral M, Loporcaro G, Scott A, Malek A (2015) Residual capacity and repairing options for reinforced concrete buildings, Research Report Prepared for the Natural Hazard Research Platform, Contract 2012-UOC-02-NHRP

Polese M, Di Ludovico M, Prota A, Manfredi G (2012) Residual capacity of earthquake damaged buildings. In: Proceedings of the 15th World Conference on Earthquake Engineering, Lisboa, Portugal

Open Access This chapter is licensed under the terms of the Creative Commons Attribution 4.0 International License (http://creativecommons.org/licenses/by/4.0/), which permits use, sharing, adaptation, distribution and reproduction in any medium or format, as long as you give appropriate credit to the original author(s) and the source, provide a link to the Creative Commons license and indicate if changes were made.

The images or other third party material in this chapter are included in the chapter's Creative Commons license, unless indicated otherwise in a credit line to the material. If material is not included in the chapter's Creative Commons license and your intended use is not permitted by statutory regulation or exceeds the permitted use, you will need to obtain permission directly from the copyright holder.

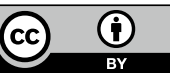

